\title{
Continuidad en la técnica de elaboración de moldes para el vaciado de plata en el área centro andina desde la época precolombina hasta la actualidad $^{1}$
}

\section{Continuity of the technique of manufacturing molds for casting silver at the Center Andean Area, from Precolumbian times}

\author{
Luisa Vetter Parodi \\ Museo Nacional Sicán; Programa de Estudios Andinos-PUCP \\ Av. Manuel Villarán 192, Dpto. 302, Urbanización Aurora, Miraflores
}

\section{RESUMEN}

En la época precolombina, los orfebres del área centro andina manejaban distintas técnicas de vaciado para elaborar piezas de metal. Una de estas técnicas era el vaciado en moldes bivalvos elaborados en arcilla y con temperantes orgánicos. Esta misma técnica de elaboración de moldes la podemos apreciar hoy en día en San Pablo de Canchis, donde los plateros cusqueños elaboran sus piezas vaciadas de plata en moldes elaborados con técnicas muy parecidas a la de los orfebres precolombinos. Para poder determinar si hubo transferencia de tecnología entre los orfebres precolombinos y los sanpablinos, hemos analizado moldes procedentes del cementerio inca de Rinconada Alta en La Molina en Lima y moldes de San Pablo de Canchis por microscopia electrónica de barrido con espectroscopia de energía dispersa (MEB-EED), fluorescencia de rayos $X(F R X)$ y difracción de rayos $X(D R X)$.

Descriptores: Plata, moldes, vaciado, época precolombina, San Pablo de Canchis, arqueometría.

\begin{abstract}
In Precolumbian times, silversmiths used different casting techniques to make their metal works. One of them was casting with bivalve molds made with clay and organic temper. The same technique can be seen nowadays at San Pablo de Canchis, where Cuzco silversmiths made their works with similar techniques to those of Precolumbian times. In order to determine if there was a technology transfer between Precolumbian silversmiths and San Pablo's, we have analized molds from the Inca cemetery of Rinconada Alta, La Molina, in Lima, and also molds from San Plablo de Canchis, using scanning electron microscope and energy dispersive spectroscopy (SEM-EDAX), $X$ ray fluorescence (XRF) and $X$ ray diffraction (XRD).
\end{abstract}

Keywords: Silver, molds, casting, Precolumbian times, San Pablo de Canchis, archeometry.

${ }^{1}$ Esta investigación forma parte de la tesis de doctorado en Historia que la autora viene realizando en el Programa de Estudios Andinos de la PUCP (2010-). 


\section{INTRODUCCIÓN}

Los plateros de San Pablo de Canchis tienen una larga tradición en la técnica del vaciado que posiblemente se inicia con los incas, cuando éstos trasladaron a orfebres ychsma de la costa central, y chimú de la costa norte al Cusco para crear piezas de oro y plata para la elite gobernante [1 y 2].

Para realizar este estudio, se ha hemos tenido acceso a moldes de arcilla para el vaciado procedentes de una excavación arqueológica en el cementerio inca de Rinconada Alta en La Molina en Lima, lugar que ocuparon los orfebres ychsma antes de su traslado al Cusco. De igual forma, viajamos aen San Pablo de Canchis en Cusco, se donde tuvoimos la posibilidad de conocer la forma de elaboración de los moldes de arcilla para la técnica del vaciado al lado de los actuales plateros sanpablinos representados por don Gregorio Cachi, mayor exponente nacional en este arte.

Con la información así obtenida, nos ha sido posible comparar las técnicas precolombinas de elaboración de moldes con las sanpablinas, las cuales se han hemos estudiado con métodos arqueométricos -microscopia electrónica de barrido con espectroscopia de energía dispersa (MEB-EED), fluorescencia de rayos $X$ (FRX) y difracción de rayos $X(D R X)-$, para determinar si hubo traspaso de conocimientos entre los plateros de la costa y de Cusco. No estamos postulando que este traspaso de conocimientos se halla mantenido sin interrupción por 500 años, sino que a pesar del tiempo transcurrido se puede observar influencias de la manufactura de moldes para la técnica del vaciado que posiblemente los plateros costeños trasladaron al Cusco en el siglo XVI [3].

\section{EL ANÁLISIS ARQUEOMÉTRICO}

Las piezas de San Pablo de Canchis fueron analizadas por diferentes técnicas y en distintos laboratorios, esto último debido a que en Lima no se cuenta con un solo Laboratorio que tenga todos los equipos necesarios para hacer un buen estudio arqueométrico [Figura 1 y 2].

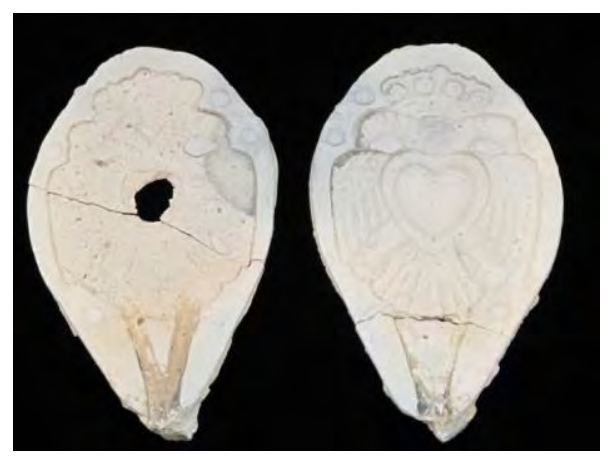

Figura 1: Molde del Águila

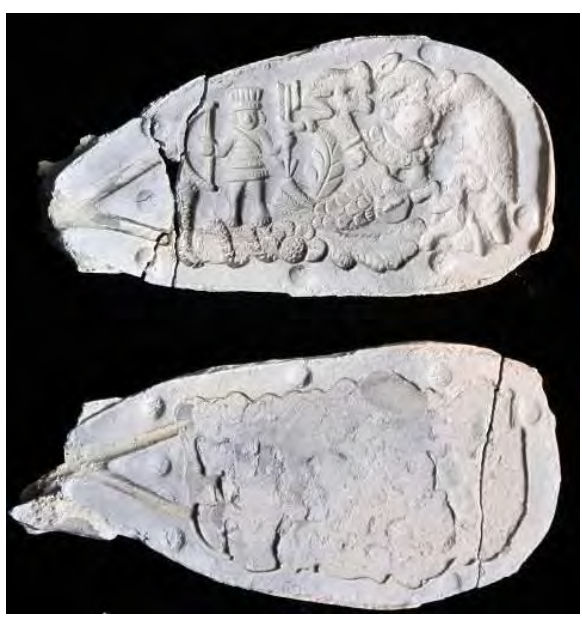

Figura 2: Molde del Nativo

En el Laboratorio de Datación de la Universidad Nacional de Ingeniería se repitió el proceso de elaboración de la masa para el modelado con la arcilla traída de San Pablo de Canchis, específicamente del cerro Pumaorcco. Se remojó la tierra de moldeo por 18 horas repitiendo lo hecho por los plateros cusqueños, luego se colocó en un mortero y se procedió a unirla (homogenizarla). Con la Cuando tuvimos una masa obtenida, se realizaronmos tres procesos:

El primero fue modelar una bola de masa sola. El resultado fue que para modelar la masa sola demoró una hora, luego de lo cual conseguimos una bola pegajosa.

Luego se modeló una bola de masa con algodón donde se demoró diez minutos y se obtuvo una bola muy modelable y lisa, además fue fácil de trabajarla.

Por último se modeló una bola de masa con lana de oveja y se demoró también diez minutos, pero la textura fue áspera, debido a la lana; además no fue tan fácil de modelar como la de algodón porque la lana es mas resistente se resiste más a que uno la doble, la manipule. 
Posteriormente se colocaron las tres muestras en una estufa a $100^{\circ} \mathrm{C}$ por diez horas y después se bajó a $50^{\circ} \mathrm{C}$ para una completa remoción del agua de humedad.

Al retirar las tres piezas de la estufa, se observó que la muestra de solo tierra para moldear, sin ningún temperante, se había rajado y cuarteado, posiblemente porque no tuvo adherencia entre partículas que permita absorber las diferencias de dilatación térmica. La pieza con el algodón y la otra con la lana de oveja no se habían rajado, conservaron la forma por efecto del temperante, es decir tenían mejor resistencia mecánica y resistencia al choque térmico. La textura de la bola que tiene algodón es más suave, más fina porque su fibra es más delgada, mientras que la de lana es una textura mucho más gruesa, más rústica. Es por esta razón que los orfebres de San Pablo de Canchis colocan este temperante orgánico para hacer que la masa sea de fácil modelado y además que soporte las altas temperaturas (resistencia en caliente) a las que se ve expuesta cuando se encuentra en la fragua para el vaciado.

La arcilla cruda o tierra de moldeo, según los resultados de DRX de muestra completa y muestra separada por decantación, es una mezcla de minerales entre los que destacan el cuarzo y las arcillas [Tabla 1].

Tabla 1: $D R X$ de la arcilla o tierra de moldeo.

\begin{tabular}{|l|l|}
\hline $\begin{array}{c}\text { Nombre del } \\
\text { mineral } \\
\text { identificado }\end{array}$ & \multicolumn{1}{|c|}{ Fórmula } \\
\hline Cuarzo & $\mathrm{SiO} 2$ \\
\hline Muscovita & $\mathrm{KAl} 2(\mathrm{Si} 3 \mathrm{Al}) \mathrm{O} 10(\mathrm{OH}, \mathrm{F}) 2$ \\
\hline Illite & $\mathrm{KAl} 2(\mathrm{Si} 3 \mathrm{Al}) \mathrm{O} 10(\mathrm{OH}, \mathrm{F}) 2$ \\
\hline Rutilo & $\mathrm{TiO} 2$ \\
\hline
\end{tabular}

Los resultados de la composición química elemental de FRX y EED para el molde del Águila, coinciden con la detección de los elementos de porcentaje mayores del $1 \%$, que es el porcentaje mínimo que hemos detectado por EED. Se encuentran dos elementos que en los resultados de EED aparecen con porcentajes mayores al 25\%: el carbono y el oxígeno; y que no aparecen por FRX debido a que la técnica no permite detectar elementos livianos. EI carbono es un elemento muy importante por cuanto evidencia la presencia de material orgánico quemado en el molde. Esta evidencia se corroboró al analizar la zona donde se encontraban las fibras de algodón en el molde del Nativo, dando un alto contenido de carbono [Tabla 2]. .

Tabla 2: EED del molde del Nativo total.

\begin{tabular}{|c|c|}
\hline Elemento & $\%$ \\
\hline Carbono & 25.46 \\
\hline Oxígeno & 41.54 \\
\hline Sodio & 1.13 \\
\hline Aluminio & 10.29 \\
\hline Sílice & 16.86 \\
\hline Azufre & 0.76 \\
\hline Potasio & 1.86 \\
\hline Calcio & 0.72 \\
\hline Hierro & 1.38 \\
\hline
\end{tabular}

La fibra orgánica, en general, cuando se quema deja cavidades que dan permeabilidad a la arcilla o tierra de moldeo, permitiendo el escape de los gases generados durante el proceso de vaciado: los gases disueltos en el metal líquido, y los gases de todo el sistema de moldeo. De esta forma, el metal líquido podrá fluir sin dificultad por la cavidad de vaciado, y además se evitará que el molde explote por la generación y confinamiento atrapamiento de los gases al interior [Figura 3].

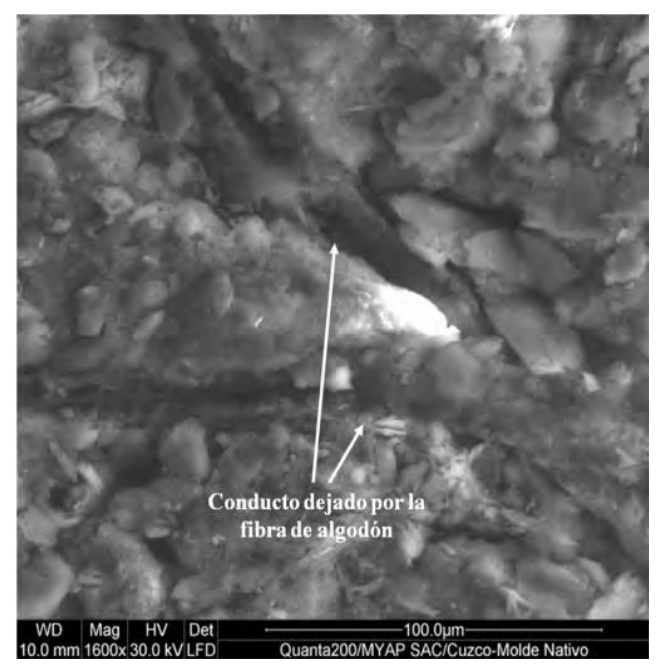

Figura 3: Evidencia de fibra orgánica en el molde del Nativo (MEB, 1600x). 
Con la elaboración de la masa en el laboratorio para medir sus propiedades mecánicas básicas, se ha constatado que la fibra es un temperante que permite darle flexibilidad a la masa posibilitando modelarla sin problemas. La diferencia entre la fibra de oveja y el algodón, es como ya se mencionó, que la primera es áspera y la segunda es fina, lo que durante el proceso de secado impide el cuarteo del molde.

La El MEB combinado con el EED hicieron han hecho posible identificar en el molde del Nativo restos de metal en la superficie del canal, los que fueron analizados en su composición, dando como resultado una composición similar de la aleación usada [Figura 4 y Tabla 3].

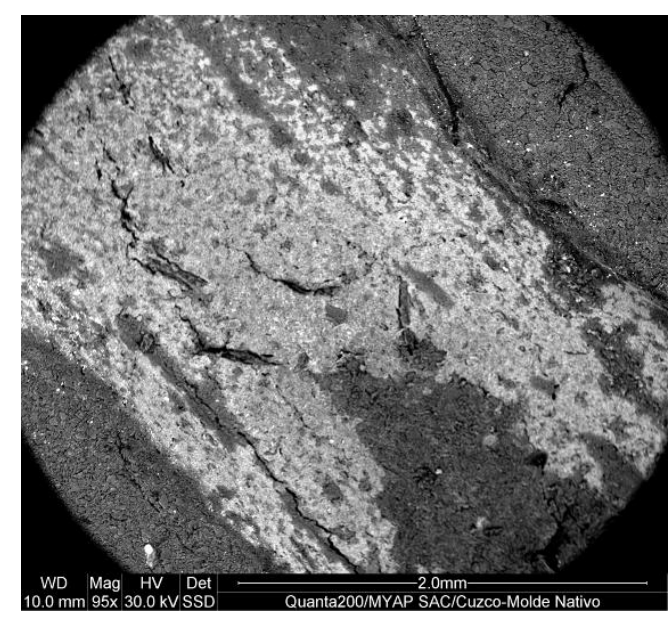

Figura 4: Canal o hito del Molde del Nativo donde se observa restos de metal adheridos a la superficie (MEB, 95x).

Tabla 3: Composición del canal o hito del molde del Nativo por EED.

\begin{tabular}{|c|c|}
\hline Elemento & $\%$ \\
\hline Oxígeno & 40.75 \\
\hline Aluminio & 8.85 \\
\hline Sílice & 18.05 \\
\hline Plata & 19.46 \\
\hline Hierro & 3.31 \\
\hline Cobre & 9.58 \\
\hline
\end{tabular}

De lo anterior, se puede podemos decir, que la arcilla que se obtiene de los cerros cercanos como el Pumaorcco es una tierra que tiene las características necesarias para ser una tierra de moldeo de buena calidad, esto sumado al uso de fibras naturales como el algodón o la lana de oveja como temperante, consigue la conformabilidad necesaria para hacer un excelente modelado. Además, las cavidades dejadas por esta fibra al quemarse el molde en la fragua, posibilitará el escape de los gases permitiendo que fluya el metal líquido y que no se quiebre el molde. Esta tierra de moldeo tiene la ventaja de tener una alta proporción de partículas de arcilla, y poca cantidad de componentes como feldespato, arena, etc.; lo que permite un buen desempeño cuando se incorpora fibra natural orgánica con las consecuencias ya estudiadas.

Se haHemos querido comparar esta técnica sanpablina del vaciado con la realizada por los orfebres precolombinos de Rinconada Alta en La Molina en Lima, para que nos permita determinar si hubo una transferencia de tecnología entre los plateros yungas de la costa central y los plateros del Cusco.

El arqueólogo Daniel Guerrero excavó un cementerio inca en la década de 1990 localizado en Rinconada Alta, donde halló que en uno de los sectores del cementerio -el Sector IIA- estaba compuesto de una serie de fardos funerarios pertenecientes a orfebres, ya que parte de su ajuar funerario estaba compuesto por herramientas de orfebrería - cinceles, punzones, buriles-, así como moldes [REFERENCIA].

Son tres moldes los que fueron hallados, uno es bivalvo y representa a un personaje con los brazos extendidos hacia los costados; el segundo es una valva que representa a un pájaro de pico largo, el tercero es un fragmento de molde donde parece que se representa una figura muy parecida al molde bivalvo [Figuras 5, 6, y 7]. 


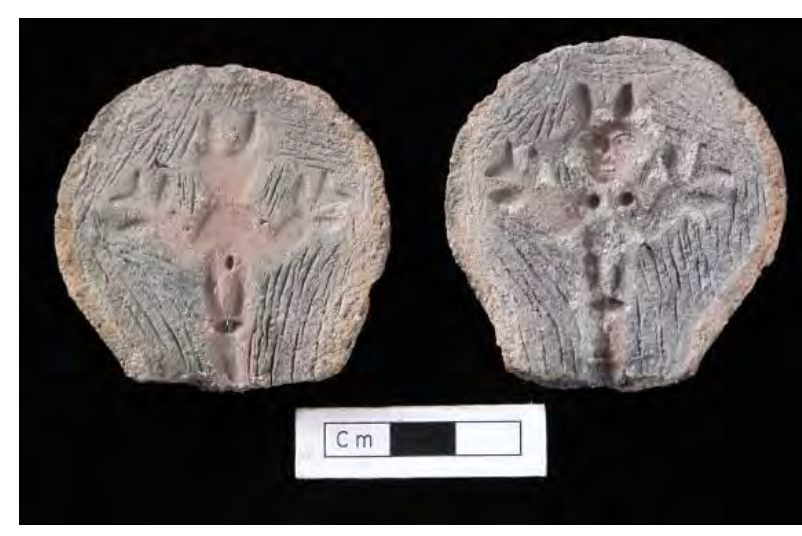

Figura 5: Molde bivalvo del Personaje.

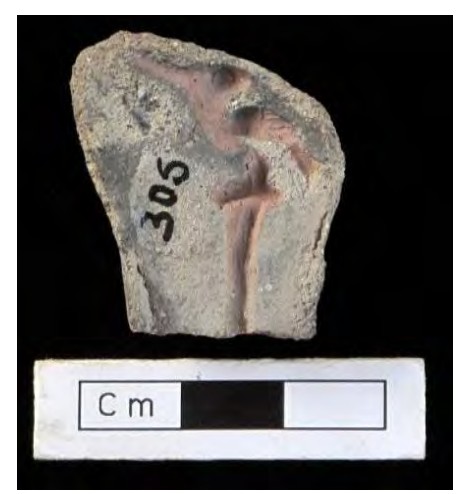

Figura 6: Molde del Pájaro.

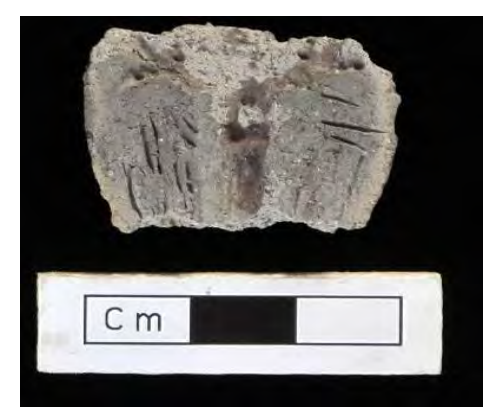

Figura 7: Fragmento de Molde.

Se han Hemos analizado estos moldes con MEBEED para comparar los resultados del análisis por FRX que se hiciera en una investigación anterior por la ingeniero Paula Olivera del IPEN [4], así como el observar microscópicamente si hay evidencia de restos de metal adheridos a la superficie del canal. Esto confirmaría o rechazaría la teoría inicial, donde se advirtió que era necesario realizar otro tipo de análisis para poder contrastar los resultados.

La ventaja con el MEB-EED es que es un método noNO destructivo. Como estos moldes son piezas arqueológicas, además existen muy pocos en el registro arqueológico y lo más importante es que provienen de una excavación científicamente controlada, los hace únicos; por tal motivo es que se debe tener el mayor cuidado posible para no dañarlos. Estas piezas se encuentran actualmente en el Museo Nacional de Arqueología, Antropología e Historia del Perú-MNAAHP.

Se ha comprobado que los tres moldes indicados han sido usados para el vaciado. Hay evidencia de que en el molde del Personaje se ha vaciado una aleación, por lo menos, ternaria de cobre-plata-oro, donde el mayor porcentaje lo tiene la plata [Tabla 4]. La composición de la aleación encontrada es similar a la usada en época precolombina para la técnica del dorado.

Tabla 4: Composición del canal en el molde del Personaje por EED.

\begin{tabular}{|c|c|}
\hline Elemento & $\%$ \\
\hline Plata & 93.04 \\
\hline Cobre & 2.78 \\
\hline Oro & 4.18 \\
\hline
\end{tabular}

En el molde del Pájaro sólo se evidenció contenido de plata, mientras que en el Fragmento de molde se halló plata y cobre. Estos resultados confirman parcialmente los resultados que se obtuvieron hace diez años la autora mediante por FRX[REF], en ese momento lanuestra conclusión fue que se habíamos logrado identificar el uso del molde del Pájaro y del Fragmento de molde, mas no el del Personaje. Se debe tomar en cuenta el alcance y límites de cada técnica de caracterización, esta vez el acceso a un MEB con EED, técnicas no destructivas y que no necesita ningún tipo de preparación de la muestra, hace que se "observeveamos" la muestra tal cual es. La imagen que nos brinda el MEB, junto con la posibilidad de determinar composiciones masivas y puntuales con el EED de la zona que se está observando, crean una herramienta fundamental para este tipo de estudios. El equipo de FRX que se usó hace diez años en el IPEN permitía analizar las piezas de manera no destructiva, pero no permitía saber dónde estaba apuntando el haz, por no contar esta técnica con una imagen microscópica de la superficie que nos guiara.

La fibra que hemos encontrado en los tres moldes es orgánica, aunque no podemos decir de qué tipo. QuizasPensamos que quizás podría ser algodón, sugiriendo y que un análisis específico de fibras debe ser llevado a cabo [Figura 8]. 


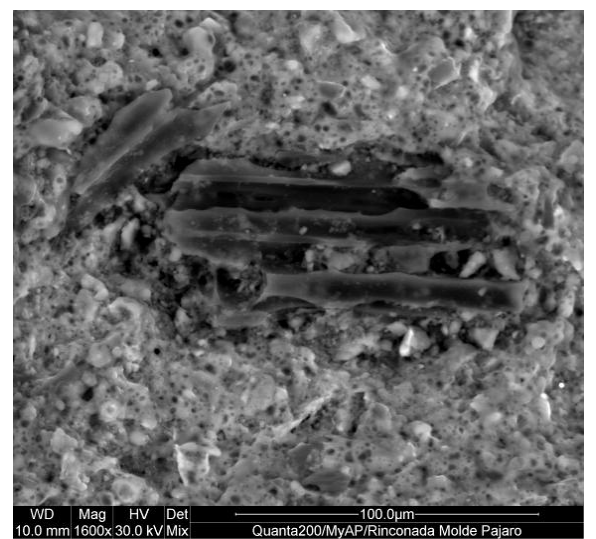

Figura 8: Evidencia de fibra orgánica en el molde del Pájaro (MEB, 1600x).

El molde del Pájaro y Fragmento de molde tienen una arcilla con una granulometría cualitativamente muy fina, mientras que la del molde del Personaje tiene una granulometría más gruesa y es sumamente heterogénea. Esta diferencia podría indicar canteras diferentes o cuidados distintos en la elaboración de la masa.

\section{RESULTADOS Y DISCUSIÓN}

Se haHemos podido confirmar que la elaboración de los moldes de Rinconada Alta en La Molina ha sido hecha colocando como temperante fibra orgánica, que creemos es algodón [ver Figura 8]. Este dato es importante porque se está usando un temperante orgánico, al igual que en Cusco [ver Figura 3], para darle flexibilidad a la masa y además conseguir una masa lisa y fácilmente modelable; y que tenga "resistencia al choque térmico".

Tanto en los moldes de Cusco como en los de Rinconada Alta, la mayor evidencia de presencia de metal se da en el hito por donde ingresa el metal al molde. El Fragmento de molde [Figura 9] resultó ser el más parecido al molde del Nativo del Cusco [ver Figura 4], porque en ambos se ve claramente la evidencia de la superficie del metal en el canal. Hay que tomar en cuenta que el Fragmento de molde ha estado enterrado por 500 años, y a pesar de eso la evidencia de su uso es totalmente clara.

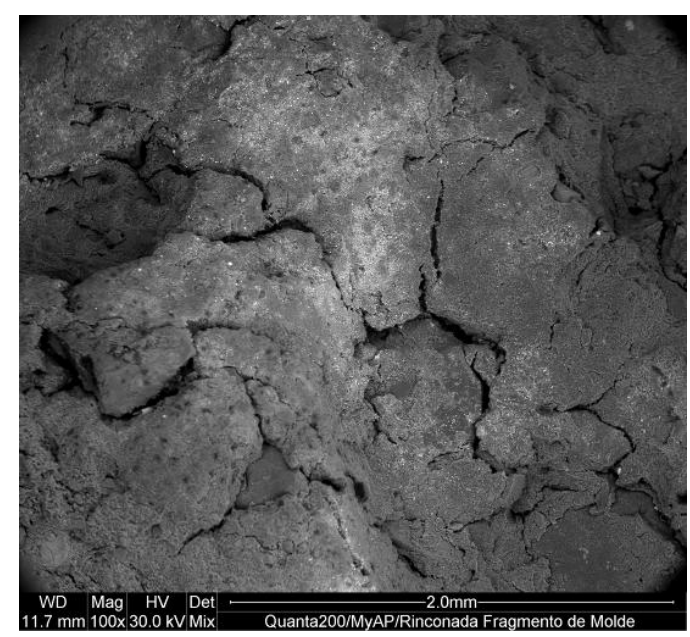

Figura 9: Canal o hito del Fragmento de Molde donde se observa restos de metal adheridos a la superficie (MEB, 100x).

Se observa, tanto en los moldes de Rinconada Alta como en los de Cusco, que hay pequeñas partículas de metal fuera de la cavidad de vaciado. Esto no indica que los orfebres de Rinconada, ni los de Cusco no supieran unir las valvas para evitar "fugas" del metal líquido, sino por el contrario, esas "fugas" han sido controladas porque sino se vería una superficie mayor de metal en la zona donde no debería de estar [Figuras 10 y 11].

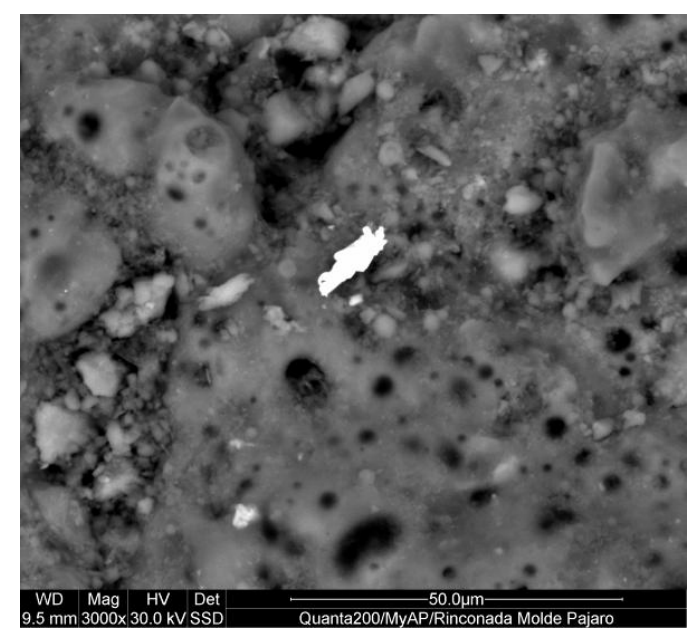

Figura 10: Evidencia de partículas de metal (zona clara) en el molde del Pájaro (MEB, 3000x). 


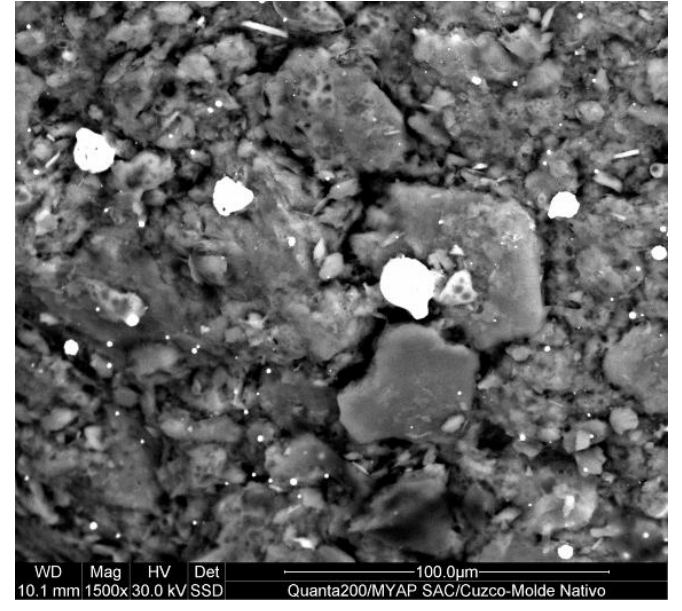

Figura 11: Evidencia de partícula de metal (zona clara) en el molde del Nativo (MEB, 1500x).

En el molde del Personaje se puede apreciar en la cara reversa unas muescas que fueron hechas para unir las dos valvas antes del vaciado, y así evitar pérdida del metal [Figura 12]. Don Gregorio al unir las dos valvas para prepararlas para el vaciado, presiona los bordes con sus dedos y utiliza las uñas para unirlas bien, dejando en algunos casos presencia de muescas en la parte reversa de las valvas [Figura 13]. Además, podemos observar que en el molde del Personaje y en el Fragmento de molde, en la cara anversa, hay líneas diagonales que pudieron haber servido para encajar unas con otras y así evitar que las valvas se muevan haciendo que el vaciado salga mal [ver Figuras 5 y 7]. Estas líneas diagonales tendrían la misma función que los círculos en alto y bajo relieve de las valvas de Cusco [ver Figuras 1 y 2].

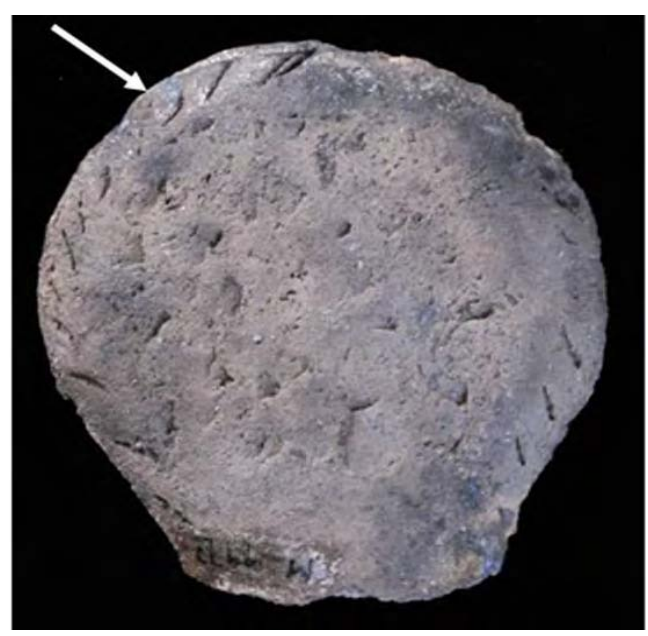

Figura 12: Evidencia de muescas (señaladas por la flecha) en el molde del Personaje.

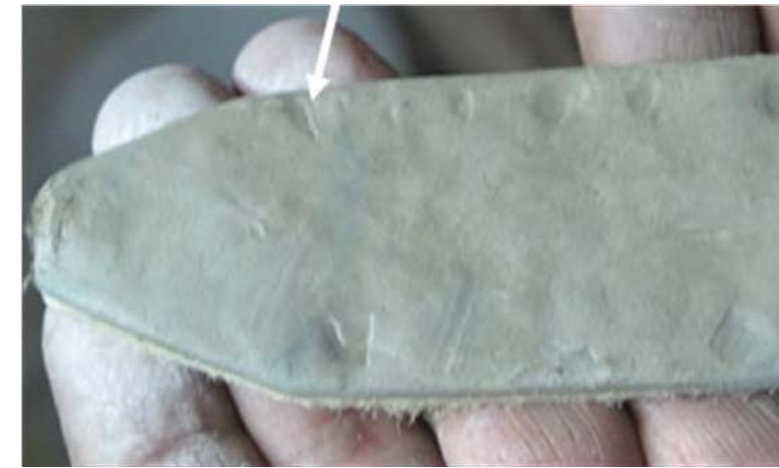

Figura 13: Evidencia de muescas (señaladas por la flecha) en el molde del Nativo.

\section{CONCLUSIONES}

Podemos decir que hay evidencias del uso de técnicas similares para el trabajo del vaciado, lo que induce a pensar que la técnica del vaciado de plata practicado por los sanpablinos fue probablemente aprendida de los plateros yungas de la costa central.

\section{AGRADECIMIENTOS}

La autora Ddeseamos expresar sunuestro agradecimiento a la familia de Gregorio Cachi Palomino, quienes nos mostraron la forma de elaboración de los moldes para la técnica del vaciado. A la Ing. Paula Olivera del Instituto Peruano de Energía Nuclear, por realizar los análisis de FRX. A la Dra. Susana Petrick del Laboratorio de Datación de la Facultad de Ciencias de la Universidad Nacional de Ingeniería - UNI por ayudarnos en el estudio de la propiedades mecánicas de la arcilla cusqueña. Al BISA-Buenaventura Ingenieros S.A., dirigido por el ingeniero Alberto Aranda por la realización de los análisis de FRX y DRX. A la Dra. Gladys Ocharán del Laboratorio de Microscopia y Aplicaciones en el Perú-MyAP por el estudio de MEB y EED. $Y$ al Dr. Christian Mesía Montenegro y conservador Luis Enrique Castillo del MNAAHP por permitirnos realizar los análisis arqueométricos a los moldes arqueológicos.

\section{REFERENCIAS}

[1] W. Espinoza. Los mitmas plateros de Ishma en el país de los ayamarca, siglos XV-XIX. En: Boletín de Lima. 30(1983)38-52, Lima.

[2] L. Vetter. Plateros indígenas en el Virreinato del Perú: siglos XVI y XVII. Fondo Editorial 
Universidad Nacional Mayor de San Marcos y Compañía de Minas Buenaventura S.A.A, Lima. 2008.

[3] L. Vetter. El platero indígena en los Andes Centrales: siglos XVI y XVII. Tesis en elaboración para optar el grado de doctor en Historia con mención en Estudios Andinos por la PUCP.

[4] P. Carcedo, L. Vetter. Instrumentos utilizados para la fabricación de piezas de metal para el período inca. 50 Congreso Internacional de Americanistas, Varsovia, Polonia. Julio, 2000. En: Baessler Archiv. 50(2002)47-66. Ethnologisches Museum de Berlín, Alemania.

[1] W. Espinoza. Los mitmas plateros de Ishma en el país de los ayamarca, siglos XV-XIX. En: Boletín de Lima. 30(1983)38-52, Lima.

[2] L. Vetter. Plateros indígenas en el Virreinato del Perú: siglos XVI y XVII. Fondo Editorial Universidad
Nacional Mayor de San Marcos y Compañía de Minas Buenaventura S.A.A, Lima. 2008.

[3] L. Vetter. El platero indígena en los Andes Centrales: siglos XVI y XVII. Tesis en elaboración para optar el grado de doctor en Historia con mención en Estudios Andinos por la PUCP.

[4] P. Carcedo, L. Vetter. Instrumentos utilizados para la fabricación de piezas de metal para el período inca. 50 Congreso Internacional de Americanistas, Varsovia, Polonia. Julio, 2000. En: Baessler Archiv. 50(2002)47-66. Ethnologisches Museum de Berlín, Alemania.

E-mail: luchivetter@hotmail.com 